\title{
A Method of Integration over Matrix Variables
}

\section{L. Mehta}

Commissariat à l'Energie Atomique, Division de la Physique, Service de Physique Théorique, CEN Saclay, F-91190 Gif-sur-Yvette, France

\begin{abstract}
The integral over two $n \times n$ hermitan matrices $Z(g, c)=\int d A d B \exp \left\{-\operatorname{tr}\left[A^{2}+B^{2}-2 c A B+\frac{g}{n}\left(A^{4}+B^{4}\right)\right]\right\}$ is evaluated in the limit of large $n$. For this purpose use is made of the theory of diffusion equation and that of orthogonal polynomials with a non-local weight. The above integral arises in the study of the planar approximation to quantum field theory.
\end{abstract}

\section{Introduction}

In their study of planar diagrams some authors $[1,3]$ have discussed integrals of the form

$$
\begin{gathered}
Z=\int \prod_{i} d M^{(i)} \exp \left\{-\sum_{i} V\left(M^{(i)}\right)+\sum_{i<j} C_{i j} \operatorname{tr} M^{(i)} M^{(j)}\right\} \\
V(M)=\operatorname{tr} M^{2}+\frac{g}{n} \operatorname{tr} M^{4}
\end{gathered}
$$

where $M^{(1)}, M^{(2)}, \ldots$ are hermitian matrices of order $n \times n$. The integral is taken over all independent real parameters entering the matrix elements,

$$
\int d M=\int_{-\infty}^{\infty} \ldots \int \prod_{i=1}^{n} d M_{i i} \prod_{1 \leqq i<j \leqq n} d\left(\operatorname{Re} M_{i j}\right) d\left(\operatorname{Im} M_{i j}\right) .
$$

The case of one matrix is the simplest. There are no cross terms containing $C_{i j}$. The integral reduces to that over the eigenvalues [4],

$$
\begin{aligned}
Z(g) & =d M \exp \left\{-\operatorname{tr} M^{2}-\frac{g}{n} \operatorname{tr} M^{4}\right\} \\
& =\text { const. } \int \exp \left\{-\sum_{i=1}^{n}\left(x_{i}^{2}+\frac{g}{n} x_{i}^{4}\right)\right\}|\Delta(X)|^{\beta} \prod^{n} d x_{i},
\end{aligned}
$$


where

$$
\Delta(X)=\prod_{1 \leqq i<j \leqq n}\left(x_{i}-x_{j}\right)
$$

and $\beta=2$. It is now known $[1,2]$ that

$$
-\frac{1}{n^{2}} \ln \frac{Z(g)}{Z(0)}=E_{0}(g)+\frac{1}{n^{2}} E_{1}(g)+0\left(n^{-4}\right)
$$

with

$$
\begin{aligned}
E_{0}(g) & =-\frac{1}{2} \ln a^{2}+\frac{1}{24}\left(a^{2}-1\right)\left(9-a^{2}\right), \\
E_{1}(g) & =\frac{1}{12} \ln \left(2-a^{2}\right), \\
a^{2} & \equiv a^{2}(g)=\frac{1}{3 \beta g}\{-1+\sqrt{1+6 \beta g}\}, \beta=2 .
\end{aligned}
$$

In stead of hermitian matrices one could have taken matrices which are real symmetric or which are quaternion self-dual. The corresponding integrals reduce again to Eq. (1.4) where the parameter $\beta$ is 1 for real symmetric matrices and it is 4 for quaternion self dual matrices. These integrals can again be evaluated in the large $n$ limit, and give the same $E_{0}(g)$ except that $\beta$ is now 1 or 4 . The correction term $E_{1}(g)$ may be different. The details of this calculation being of no interest are omitted.

The next difficult case of two matrices was discussed by Itzykson and Zuber [3]. They reduced the integral to that over the eigenvalues. However, the expressions given by them are too complicated. Below we will reinvestigate this case

$$
Z(g, c)=\int d A d B \exp \left\{-\operatorname{tr}\left(A^{2}+B^{2}\right)-\frac{g}{n} \operatorname{tr}\left(A^{4}+B^{4}\right)+2 c \operatorname{tr} A B\right\}
$$

where $A$ and $B$ are $n \times n$ hermitian matrices. We will show that

$$
\frac{1}{n^{2}} \ln \frac{Z(g, c)}{Z(0, c)}=\int_{0}^{1}(1-x)\left\{\ln f(x)-\ln \frac{c x}{2\left(1-c^{2}\right)}\right\} d x+0\left(n^{-2}\right)
$$

where $f(x)$ is given by an algebraic equation of the fifth degree

$$
f(x)\left\{\left(1-6 \frac{g}{c} f(x)\right)^{-2}-c^{2}\right\}+12 g^{2} f^{3}(x)-\frac{1}{2} c x=0,
$$

and the root to be taken equals $\frac{1}{2} c x\left(1-c^{2}\right)^{-1}$ when $g=0$.

\section{The Method of Diffusion Equation}

Consider the partial differential equation

$$
\frac{\partial \xi}{\partial t}=\frac{1}{2} \sum_{i} D_{i} \frac{\partial^{2} \xi}{\partial x_{i}^{2}}
$$


where the constants $D_{i}$ may be unequal for different directions. The unique solution satisfying the initial condition $\xi(X ; 0)=\eta(X)$ is known to be [5]

$$
\begin{gathered}
\xi(X ; t)=\int K(X, Y ; t) \eta(Y) d Y, \\
K(X, Y ; t)=\prod_{i}\left(2 \pi D_{i} t\right)^{-1 / 2} \exp \left\{-\sum_{i}\left(x_{i}-y_{i}\right)^{2} /\left(2 D_{i} t\right)\right\} .
\end{gathered}
$$

Now let $A$ be an $n \times n$ hermitian matrix with elements $A_{i j}$. The $A_{i i}$ are real, while the real and imaginary parts of $A_{i j}$ for $i<j$ are denoted by $\operatorname{Re} A_{i j}$ and $\operatorname{Im} A_{i j}$ respectively. Similarly for the matrix $B$. Then

$$
\begin{gathered}
\xi(A ; t)=\int K(A, B ; t) \eta(B) d B \\
K(A, B ; t)=(2 \pi t)^{-n^{2} / 2} \exp \left\{-\frac{1}{2 t} \operatorname{tr}(A-B)^{2}\right\} \\
\equiv(2 \pi t)^{-n^{2} / 2} \exp \left\{-\frac{1}{2 t}\left[\sum_{i}\left(A_{i i}-B_{i i}\right)^{2}+2 \sum_{i<j}\left(\operatorname{Re} A_{i j}-\operatorname{Re} B_{i j}\right)^{2}\right.\right. \\
\left.\left.+2 \sum_{i<j}\left(\operatorname{Im} A_{i j}-\operatorname{Im} B_{i j}\right)^{2}\right]\right\} \\
\int d B \equiv \int_{-\infty}^{\infty} \ldots \int \prod_{i} d B_{i i} \prod_{i<j} d\left(\operatorname{Re} B_{i j}\right) d\left(\operatorname{Im} B_{i j}\right)
\end{gathered}
$$

satisfies the equation

$$
\begin{aligned}
& \frac{\partial \xi}{\partial t}=\frac{1}{2} \nabla_{A}^{2} \xi \\
& \nabla_{A}^{2}=\sum_{i} \frac{\partial^{2}}{\partial A_{i i}^{2}}+\frac{1}{2} \sum_{i<j}\left\{\frac{\partial^{2}}{\partial\left(\operatorname{Re} A_{i j}\right)^{2}}+\frac{\partial^{2}}{\partial\left(\operatorname{Im} A_{i j}\right)^{2}}\right\}
\end{aligned}
$$

and the initial condition

$$
\xi(A ; 0)=\eta(A) .
$$

As $A$ and $B$ are hermitian, we can choose unitary matrices $U_{A}$ and $U_{B}$ such that

$$
A=U_{A}^{+} X U_{A}, \quad B=U_{B}^{+} Y U_{B},
$$

where $X=\left[x_{i} \delta_{i j}\right]$ and $Y=\left[y_{i} \delta_{i j}\right]$ are diagonal matrices. The $x_{i}$ are the eigenvalues of $A$ and the $y_{i}$ are those of $B$. Changing the variables from matrix elements to the $n$ eigenvalues and $n(n-1)$ angle parameters on which $U_{A}$ and $U_{B}$ depend, we have [4]

$$
d B=\Delta^{2}(Y) d Y d \Omega_{B}, \quad d Y=\prod_{i=1}^{n} d y_{i}
$$


so that

$$
\begin{aligned}
\xi(A ; t) & =\xi\left(X, \Omega_{A} ; t\right) \\
& =(2 \pi t)^{-n^{2} / 2} \int \exp \left\{-\frac{1}{2 t} \operatorname{tr}\left(X-U^{+} Y U\right)^{2}\right\} \eta\left(Y, \Omega_{B}\right) \Delta^{2}(Y) d Y d \Omega_{B},
\end{aligned}
$$

where

$$
U=U_{B} U_{A}^{+}
$$

Observe that if $\eta(B)$ is independent of $\Omega_{B}$, then $\xi(A ; t)$ is also independent of $\Omega_{A}$ as can be seen by a change of variables from $\Omega_{B}$ to $\Omega$ (depending on $U$ ),

$$
\xi(X ; t)=\text { const. } t^{-n^{2} / 2} \int \exp \left\{-\frac{1}{2 t} \operatorname{tr}\left(X-U^{+} Y U\right)^{2}\right\} \eta(Y) \Delta^{2}(Y) d Y d \Omega .
$$

Seperating ${ }^{1}$ the Laplacian into parts depending on $X$ and on $U_{A}$,

$$
\nabla_{A}^{2}=\frac{1}{\Delta^{2}(X)} \sum_{i} \frac{\partial}{\partial x_{i}} \Delta^{2}(X) \frac{\partial}{\partial x_{i}}+\nabla_{\Omega_{A}}^{2}
$$

one sees that $\xi(X ; t)$ satisfies the (diffusion) equation

$$
\frac{\partial \xi}{\partial t}=\frac{1}{2} \frac{1}{\Delta^{2}(X)} \sum_{i} \partial_{i} \Delta^{2}(X) \partial_{i} \xi, \quad \partial_{i} \equiv \frac{\partial}{\partial x_{i}},
$$

and has the initial value

$$
\xi(X ; 0)=\eta(X)
$$

Set

$$
F(X ; t)=\Delta(X) \xi(X ; t)
$$

Then

$$
\begin{aligned}
\sum_{i} \partial_{i}^{2} F & =\sum_{i}\left\{\Delta \partial_{i}^{2} \xi+2\left(\partial_{i} \Delta\right)\left(\partial_{i} \xi\right)+\xi \partial_{i}^{2} \Delta\right\} \\
& =\Delta(X)\left\{\frac{1}{\Delta^{2}(X)} \sum_{i} \partial_{i}\left(\Delta^{2}(X) \partial_{i} \xi\right)\right\}
\end{aligned}
$$

Thus $F(X ; t)$ satisfies the (diffusion) equation

$$
\frac{\partial F}{\partial t}=\frac{1}{2} \sum_{i} \frac{\partial^{2} F}{\partial x_{i}^{2}}
$$

and has the initial value

$$
F(X ; 0)=\Delta(X) \xi(X ; 0)=\Delta(X) \eta(X) .
$$

1 The Jacobian of the transformation from matrix elements to the eigenvalues and angle variables for a hermitian matrix is $\Delta^{2}(X) f\left(\Omega_{A}\right)$, where $f$ is independent of the $x_{i}[4]$. Therefore the Laplacian is given by Eq. (2.15) ([5], end of Chap. 1) 
Therefore $F(X ; t)$ is given by [5]

$$
F(X ; t)=\text { const. } t^{-n / 2} \int \exp \left\{-\frac{1}{2 t} \sum_{i=1}^{n}\left(x_{i}-y_{i}\right)^{2}\right\} \Delta(Y) \eta(Y) d Y .
$$

choose

$$
\eta(B)=\exp \left\{-V(B)+c \operatorname{tr} B^{2}\right\}=\exp \left\{-V(Y)+c \sum_{i} y_{i}^{2}\right\},
$$

where $V(M)$ depends only on the eigenvalues of $M$. Setting $c=\frac{1}{2 t}$ in Eqs. (2.4)-(2.6), (2.18) and (2.22) one gets

$$
\begin{aligned}
& \Delta(X) \int d B \exp \left\{-V(B)+c \operatorname{tr} B^{2}-c \operatorname{tr}(A-B)^{2}\right\} \\
& \quad=\left(\frac{\pi}{2 c}\right)^{n(n-1) / 2} \int d Y \exp \left\{-V(Y)+c \sum_{i} y_{i}^{2}-c \sum_{i}\left(x_{i}-y_{i}\right)^{2}\right\} \Delta(Y) .
\end{aligned}
$$

The constant is obtained by choosing $V(B)=c \operatorname{tr} B^{2}$ and performing the gaussian integrals on both sides. Therefore

$$
\begin{aligned}
& \int \exp \{-V(A)-V(B)+2 c \operatorname{tr} A B\} d A d B \\
&= \text { const. } \int d X d \Omega_{A} \Delta^{2}(X) \exp \left\{-V(X)+c \sum_{i} x_{i}^{2}\right\} \\
& \cdot \int d B \exp \left\{-V(B)+c \operatorname{tr} B^{2}-c \operatorname{tr}(A-B)^{2}\right\} \\
&= \text { const. } \int d X d Y \Delta(X) \Delta(Y) \exp \left\{-V(X)-V(Y)+2 c \sum_{i} x_{i} y_{i}\right\} .
\end{aligned}
$$

This is essentially the result of Itzykson-Zubar [3] expressed in a simpler form. The constant can be fixed by considering $V(A)=\operatorname{tr} A^{2}=\sum x_{i}^{2}$. The gaussian integral on the left hand side is then straight forward, while that on the right hand side is given in the appendix. As a result the unknown constant is

$$
\pi^{n(n-1)}(2 c)^{-(1 / 2) n(n-1)}\left(\prod_{1}^{n} i !\right)^{-1}
$$

\section{Orthogonal Polynomials Revisited}

To get the asymptotic behavior of $Z(g, c) / Z(0, c)$.

where

$$
\begin{aligned}
Z(g, c)= & \int \exp \left\{-\operatorname{tr}\left(A^{2}+B^{2}\right)-\frac{g}{n} \operatorname{tr}\left(A^{4}+B^{4}\right)+2 c \operatorname{tr} A B\right\} d A d B \\
= & \text { const. } \int \exp \left\{-\sum_{i}\left(x_{i}^{2}+y_{i}^{2}\right)-\frac{g}{n} \sum_{i}\left(x_{i}^{4}+y_{i}^{4}\right)+2 c \sum_{i} x_{i} y_{i}\right\} \\
& \cdot \Delta(X) \Delta(Y) \prod_{i} d x_{i} d y_{i},
\end{aligned}
$$

we will use orthogonal polynomials with a non-local weight. 
Writting $\Delta(X)=\prod_{i<j}\left(x_{i}-x_{j}\right)$ as the Vandermonde determinant, one sees that

$$
\Delta(X)=\operatorname{det}\left[x_{i}^{j-1}\right]=\operatorname{det}\left[P_{j-1}\left(x_{i}\right)\right]_{i, j=1,2, \ldots, n}
$$

where

$$
P_{j}(x)=x^{j}+\sum_{0}^{j-1} a_{k} x^{k}
$$

is an arbitrary polynomial of degree $j$ with the coefficient of $x^{j}$ equal to 1 . Similarly,

$$
\Delta(Y)=\operatorname{det}\left[Q_{j-1}\left(y_{i}\right)\right]_{i, j=1,2, \ldots, n,}
$$

where $Q_{j}(x)$ is another set of similar polynomials.

Since

$$
w(x, y)=\exp \left\{-\left(x^{2}+y^{2}\right)-\frac{g}{n}\left(x^{4}+y^{4}\right)+2 c x y\right\}
$$

is symmetric in $x$ and $y$, we will choose $P_{i}(x)=Q_{i}(x)$ and such that

$$
\iint_{-\infty}^{\infty} w(x, y) P_{i}(x) P_{j}(y) d x d y=h_{i} \delta_{i j}
$$

where the Kronecker symbol $\delta_{i j}$ is 1 or 0 according as $i=j$ or $i \neq j$. Such a choice is possible. In fact

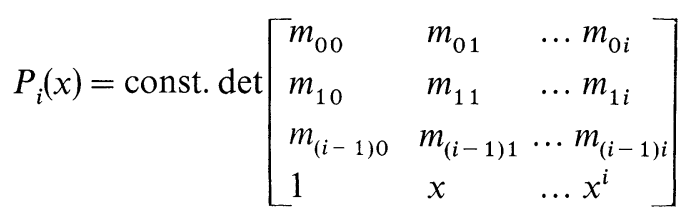

where

$$
m_{i j}=\int_{-\infty}^{\infty} \int w(x, y) x^{i} y^{j} d x d y
$$

are the moments of $w(x, y)$. In particular, since $y^{j}$ can be expressed as a linear combination of $P_{k}(y)$ with $k \leqq j$, one has

$$
\iint_{-\infty}^{\infty} w(x, y) P_{i}(x) y^{j} d x d y=0, \text { for } i>j
$$

With such a choice of $P_{i}(x)$ we expand the two Vandermonde determinants, multiply and use the orthogonal property (3.6) to integrate various products. The only terms which contribute have equal indices of the polynomials in $x$ and in $y$, they contribute the same quantity, and they are $n$ ! in number. Thus

$$
Z(g, c)=\text { const. } n ! \prod_{0}^{n-1} h_{j}(g, c),
$$

and we need to know the asymptotic behaviour of the product of $h_{j}$. For this purpose, we proceed as with the usual orthogonal polynomials. 
As $w(-x,-y)=w(x, y), m_{i j}=0$ for $i+j$ odd, and $P_{i}(x)$ has a definite parity,

Let

$$
P_{i}(-x)=(-1)^{i} P_{i}(x)
$$

$$
x P_{i}(x)=P_{i+1}(x)+R_{i} P_{i-1}(x)+S_{i} P_{i-3}(x),
$$

where $R_{i}$ and $S_{i}$ are certain coefficients. Iterating thrice we get

$$
\begin{aligned}
x^{3} P_{i}(x)= & P_{i+3}(x)+\left(R_{i}+R_{i+1}+R_{i+2}\right) P_{i+1}(x) \\
& +\left\{R_{i}\left(R_{i-1}+R_{i}+R_{i+1}\right)+\left(S_{i}+S_{i+1}+S_{i+2}\right)\right\} P_{i-1}(x)+\ldots .
\end{aligned}
$$

Thus expressing $x^{k} P_{i}(x)$ as linear combinations of $P_{j}(x), j \leqq i+k$, and using equation $(3,6)$ we get

$$
\begin{gathered}
\iint_{-\infty}^{\infty} P_{i-1}(x) P_{i}(y)\left(x-c y+2 \frac{g}{n} x^{3}\right) w(x, y) d x d y \\
=h_{i}\left\{1+\frac{2 g}{n}\left(R_{i-1}+R_{i}+R_{i+1}\right)\right\}-c R_{i} h_{i-1} .
\end{gathered}
$$

Also integrating on $x$ by parts, the left hand side of the above equation is

$$
\frac{1}{2} \iint_{-\infty}^{\infty} \frac{d P_{i-1}(x)}{d x} P_{i}(y) w(x, y) d x d y=0,
$$

because of equation (3.9). From the last two equations we get

$$
h_{i}\left\{1+\frac{2 g}{n}\left(R_{i-1}+R_{i}+R_{i+1}\right)\right\}=c R_{i} h_{i-1} .
$$

Similarly by integrating

$$
P_{i}(x) P_{i-1}(y)\left(x-c y+\frac{2 g}{n} x^{3}\right) w(x, y)
$$

and

$$
P_{i-3}(x) P_{i}(y)\left(x-c y+\frac{2 g}{n} x^{3}\right) w(x, y)
$$

in two different ways, we get the relations

$$
c h_{i}=h_{i-1}\left\{-\frac{i}{2}+R_{i}\left[1+\frac{2 g}{n}\left(R_{i-1}+R_{i}+R_{i+1}\right)\right]+\frac{2 g}{n}\left(S_{i}+S_{i+1}+S_{i+2}\right)\right\} \text {, }
$$

and

$$
2 \frac{g}{n} h_{i}=c S_{i} h_{i-3}
$$

\section{Asymptotic Evaluation of $Z(g, c)$}

Let us write $f_{i}=h_{i} / h_{i-1}$, so that Eqs. (3.16), (3.19) and (3.20) can be rewritten as 


$$
\begin{aligned}
f_{i}= & c R_{i}\left\{1+\frac{2 g}{n}\left(R_{i-1}+R_{i}+R_{i+1}\right)\right\}^{-1}, \\
c f_{i}= & -\frac{i}{2}+R_{i}\left\{1+\frac{2 g}{n}\left(R_{i-1}+R_{i}+R_{i+1}\right)\right\} \\
& +\frac{2 g}{n}\left(S_{i}+S_{i+1}+S_{i-2}\right),
\end{aligned}
$$

and

$$
c S_{i}=\frac{2 g}{n} f_{i} f_{i-1} f_{i-2}
$$
Thus

For large $i$ and $n$, the $f_{i}, R_{i}$ and $S_{i}$ can be replaced by continuous functions.

$$
\begin{array}{rlrl}
f_{i} & \sim n f(x), & f_{i \pm 1} & \sim n f(x \pm \varepsilon), \\
R_{i} \sim n R(x), & R_{i \pm 1} & \sim n R(x \pm \varepsilon), \\
S_{i} \sim n^{2} S(x), & S_{i \pm 1} & \sim n^{2} S(x \pm \varepsilon), \\
x=\frac{i}{n}, & \varepsilon=\frac{1}{n} .
\end{array}
$$

Making these substitutions, we get to the leading order,

$$
\begin{aligned}
f(x) & =c R(x)\{1+6 g R(x)\}^{-1}, \\
c f(x) & =-\frac{x}{2}+R(x)(1+6 g R(x)+6 g S(x), \\
c S(x) & =2 g f^{3}(x) .
\end{aligned}
$$

Eliminating $R(x)$ and $S(x)$ from the last three equations, one gets

$$
f(x)\left\{\left(1-6 \frac{g}{c} f(x)\right)^{-2}-c^{2}\right\}+12 g^{2} f^{3}(x)=\frac{1}{2} c x .
$$

When $g=0$, the value of $f(x)$ will be denoted by $f_{0}(x)$. From (4.6) and (4.7)

$$
f_{0}(x)=\frac{1}{2} c x\left(1-c^{2}\right)^{-1} \text {. }
$$

Now from Eq. (3.10) we have

$$
\begin{gathered}
\ln \frac{Z(g, c)}{Z(0, c)}=\sum_{i=0}^{n-1} \ln \frac{h_{i}(g, c)}{h_{i}(0, c)}, \\
\sum_{i=0}^{n-1} \ln h_{i}=n \ln h_{0}+\sum_{i=1}^{n}(n-i) \ln f_{i}, \\
\frac{1}{n^{2}} \sum_{i=1}^{n}(n-i) \ln f_{i}(g, c)=\int_{0}^{1}(1-x) \ln (n f(x)) d x+0\left(n^{-2}\right) .
\end{gathered}
$$


Therefore

$$
\frac{1}{n^{2}} \ln \frac{Z(g, c)}{Z(0, c)}=\frac{1}{n} \ln \frac{h_{0}(g, c)}{h_{0}(0, c)}+\int_{0}^{1}(1-x) \ln \frac{f(x)}{f_{0}(x)} d x+0\left(n^{-2}\right) .
$$

But

$$
\begin{aligned}
h_{0}(g, c) & =\iint_{-\infty}^{\infty} d x d y \exp \left[-\left\{x^{2}+y^{2}-2 c x y+\frac{g}{n}\left(x^{4}+y^{4}\right)\right\}\right] \\
& =\frac{\pi}{\sqrt{1-c^{2}}}\left\{1-\frac{3}{2} \frac{g}{n}\left(1-c^{2}\right)^{-2}+0\left(n^{-2}\right)\right\}
\end{aligned}
$$

Hence

$$
\ln \frac{h_{0}(g, c)}{h_{0}(0, c)}=0\left(n^{-1}\right) .
$$

Eqs. (4.14), (4.16), (4.9) and (4.10) together give the result announced in the introduction, Eqs. (1.11) and (1.12).

\section{Some Remarks}

5.1. Formula (2.25) looks trivial, but it is not. To be honest, we have no shorter way to derive it.

Itzykson and Zuber [3] derive a formula equivalent to (2.25) in a different way as well. They introduce the decomposition of unity into characters of irreducible representations of the unitary group. Using the orthogonality of these characters they can perform the angular integrations. The final result is a series containing eigenvalues of $A$ and $B$, characters of irreducible representations of the unitary group, their dimensions and the number of times an irreducible representation occurs in various Kronecker powers of the initial matrix. This method can be adapted to deal with real symmetric or quaternion self-dual matrices; one has only to replace the unitary group by the orthogonal or the symplectic group. The formulas however, do not seem to be simple.

5.2. The same method adapted to evaluate the integral over a chain of matrices

$$
\int \exp \left\{-\sum_{i=1}^{j} V\left(M^{(i)}\right)+2 \sum_{i=1}^{j-1} c_{i} M^{(i)} M^{(i+1)}\right\} \prod_{1}^{j} d M^{(i)}
$$

in the limit of large $n$ will be considered elsewhere [7]

5.3. An expansion in powers of $g$ gives

$$
\frac{f(x)}{f_{0}(x)}=1-6 g x\left(1-c^{2}\right)^{-2}+3 g^{2} x^{2}\left(1-c^{2}\right)^{-4}\left(c^{4}+8 c^{2}+15\right)+0\left(g^{3}\right)
$$

so that

$$
\begin{aligned}
-E_{0}(g) & =\int_{0}^{1}(1-x) \ln \frac{f(x)}{f_{0}(x)} d x \\
& =-g\left(1-c^{2}\right)^{-2}+\frac{1}{4} g^{2}\left(1-c^{2}\right)^{-4}\left(c^{4}+8 c^{2}+9\right)+0\left(g^{3}\right)
\end{aligned}
$$


5.4. Denoting by $\langle>$ the average with respect to any positive measure, one has the inequality [16].

$$
\left\langle e^{F}\right\rangle \geqq e^{\langle F\rangle}
$$

Taking

$$
\langle\ldots\rangle=\int d A d B \exp \left\{-\operatorname{tr}\left(A^{2}+B^{2}-2 c A B\right)\right\} \ldots,
$$

or

$$
\langle\ldots\rangle \equiv \int d X d Y \Delta(X) \Delta(Y) \exp \left\{-\sum_{1}^{b}\left(x_{i}^{2}+y_{i}^{2}-2 c x_{i} y_{i}\right)\right\} \ldots
$$

and

$$
F=-\frac{g}{n} \operatorname{tr}\left(A^{4}+B^{4}\right)=-\frac{g}{n} \sum_{1}^{n}\left(x_{i}^{4}+y_{i}^{4}\right),
$$

we get the inequality (see the appendix)

$$
\begin{aligned}
\frac{Z(g, c)}{Z(0, c)} & =\exp \left\{-n^{2} E_{0}(g)-E_{1}(g)+0\left(n^{-2}\right)\right\} \\
& \geqq \exp \left\{-g\left(1-c^{2}\right)^{-2}\left(n^{2}+\frac{1}{2}\right)\right\}
\end{aligned}
$$

Thus one sees that in agreement with Eq. (5.2),

$$
\begin{aligned}
& E_{0}(g)=+g\left(1-c^{2}\right)^{-2}+0\left(g^{2}\right), \\
& E_{1}(g)=+\frac{1}{2} g\left(1-c^{2}\right)^{-2}+0\left(g^{2}\right) .
\end{aligned}
$$

In general, let $D$ be the $p \times p$ matrix $\left[\delta_{i j}-C_{i j}\right]$ and $D_{k}$ the same matrix with its $k^{\text {th }}$ row and $k^{\text {th }}$ column removed. Observe that

$$
\begin{aligned}
\int \exp & \left\{-\sum_{i=1}^{p} \operatorname{tr} A_{i}^{2}+2 \sum_{1 \leqq i<j \leqq p} C_{i j} \operatorname{tr} A_{i} A_{j}\right\} d A_{1} \ldots d A_{k-1} d A_{k+1} \ldots d A_{p} \\
= & \left(2^{n}\left(\frac{\pi}{2}\right)^{n^{2}}\right)^{(p-1) / 2} a_{k} \exp \left(-b_{k} \operatorname{tr} A_{k}^{2}\right),
\end{aligned}
$$

with

$$
a_{k}=\left(\operatorname{det} D_{k}\right)^{-n^{2} / 2}, \quad a_{k} b_{k}^{-n^{2} / 2}=(\operatorname{det} D)^{-n^{2} / 2} .
$$

A power series expansion in $g$ gives

$$
\begin{aligned}
Z(g) & \equiv \int \exp \left\{-\sum_{i=1}^{p} \operatorname{tr}\left(A_{i}^{2}+\frac{g}{n} A_{i}^{4}\right)+2 \sum_{1 \leqq i<j \leqq p} C_{i j} \operatorname{tr} A_{i} A_{j}\right\} d A_{1} \ldots d A_{p} \\
& =Z(0)\left\{1-\frac{g}{n} \sum_{k=1}^{p} \int d A_{k} \operatorname{tr} A_{k}^{4} \exp \left(-b_{k} \operatorname{tr} A_{k}^{2}\right) / \int d A_{k} \exp \left(-b_{k} \operatorname{tr} A_{k}^{2}\right)+\ldots\right\} \\
& =Z(0)\left\{1-\frac{g}{4}\left(2 n^{2}+1\right) \sum_{k=1}^{p} b_{k}^{-2}+0\left(g^{2}\right)\right\} .
\end{aligned}
$$


So that

$$
\frac{Z(g)}{Z(0)} \geqq \exp \left\{-\frac{1}{4} g\left(2 n^{2}+1\right) \sum_{k=1}^{p}\left(D^{-1}\right)_{k k}^{2}\right\}
$$

5.5. An obvious upper bound for $Z(g) / Z(0)$ is 1 . Another of the form $k_{1} g^{k_{2}}$ can be obtained by Schwartz's inequality.

5.6. Note that if $V(M)$ has a term in $\operatorname{tr} M^{6}\left(\operatorname{tr} M^{8}, \ldots\right)$, then $x P_{i}(x)$ in Eq. (3.12) will also have a $P_{i-5}(x)\left(P_{i-7}(x), \ldots\right)$ present.

5.7. Let us represent the integral in Eq. (1.1) by a graph; the matrices $M^{(i)}$ are noted as points and the points $i$ and $j$ are joined by a line if $C_{i j} \neq 0$. If this graph contains no cycles, the angle variables can be integrated by using Eq. (2.24). The remaining integrations over the eigenvalues, even in the limit of large $n$, are not simple.

5.8. Examples. For a cyclic graph with $p$ points,

$$
Z(g)=\int \prod_{1}^{p} d A_{i} \exp \left\{-\sum_{i=1}^{p} V\left(A_{i}\right)+2 c \sum_{i=1}^{p} \operatorname{tr} A_{i} A_{i+1}\right\},
$$

$A_{p+1} \equiv A_{1}$, with $V$ as in Eq. (1.2).

$$
\begin{gathered}
\left(D^{-1}\right)_{k k}=\frac{1}{p} \sum_{j=1}^{p}\left(1-2 c \cos \frac{2 \pi j}{p}\right)^{-1}=\left(\operatorname{th} \theta \operatorname{th} \frac{p \theta}{2}\right)^{-1}, \\
Z(g) \geqq Z(0) \cdot \exp \left\{-\frac{g}{4}\left(2 n^{2}+1\right) p\left(\operatorname{th} \theta \operatorname{th} \frac{p \theta}{2}\right)^{-2}\right\},
\end{gathered}
$$

where $2 c \operatorname{ch} \theta=1$.

For a $p \times q$ square lattice graph with periodic boundary conditions,

$$
\begin{aligned}
Z(g)= & \int \prod_{i=1}^{p} \prod_{j=1}^{q} d A_{i j} \exp \cdot\left\{-\sum_{i=1}^{p} \sum_{j=1}^{q}\left[V\left(A_{i j}\right)-2 C_{1} \operatorname{tr} A_{i j} A_{(i+1) j}\right.\right. \\
& \left.\left.-2 C_{2} \operatorname{tr} A_{i j} A_{i(j+1)}\right]\right\}, \\
A_{i(q+1)} \equiv & A_{i 1}, \quad A_{(p+1) j} \equiv A_{1 j}, \quad V(A)=\operatorname{Tr} A^{2}+\frac{g}{n} \operatorname{tr} A^{4} . \\
\left(D^{-1}\right)_{i j, i j}= & \frac{1}{p q} \sum_{i=1}^{p} \sum_{j=1}^{q}\left(1-2 C_{1} \cos \frac{2 \pi i}{p}-2 C_{2} \cos \frac{2 \pi j}{q}\right)^{-1} \\
\approx & \frac{1}{4 \pi^{2}} \int_{0}^{2 \pi}\left(1-2 C_{1} \cos \theta-2 C_{2} \cos \phi\right)^{-1} d \theta d \phi \\
= & \frac{1}{\pi} \int_{0}^{\pi}\left\{\left(1-2 C_{1} \cos \theta\right)^{2}-4 C_{2}^{2}\right\}^{-1 / 2} d \theta
\end{aligned}
$$

for $p$ and $q$ very large. Hence

$$
\frac{Z(g)}{Z(0)} \geqq \exp \left[-\frac{g^{2}}{\pi^{2}}\left(2 n^{2}+1\right) p q\left\{1-4\left(C_{1}-C_{2}\right)^{2}\right\}^{-1}\left\{\int_{0}^{\pi / 2}\left(1-\alpha^{2} \sin ^{2} \theta\right)^{-1 / 2} d \theta\right\}^{2}\right],
$$


with $\alpha^{2}=16 C_{1} C_{2}\left\{1-4\left(C_{1}-C_{2}\right)^{2}\right\}^{-1}$.

For a star graph with $\mathrm{m}$ branches

$$
\begin{aligned}
Z(g)= & \int d A \prod_{1}^{m} d B_{i} \exp \left\{-V(A)-\sum_{1}^{m} V\left(B_{i}\right)+2 c \operatorname{tr} \sum_{1}^{m} A B_{i}\right\}, \\
\frac{Z(g)}{Z(0)}= & \left(1-m c^{2}\right)^{(1 / 2) n^{2}}\left(2 c^{-m}\right)^{(1 / 2) n(n-1)} \pi^{-(1 / 2) n}\left(\prod_{1}^{n} j !\right)^{-1} . \\
& \cdot \int \exp \left\{-V(X)+m c^{2} X^{2}\right\}\left\{\operatorname{det}\left[F_{j-1}\left(x_{k}\right)\right] / \Delta(X)\right\}^{m} \Delta^{2}(X) d X \\
\geqq & \exp \left[-\frac{g}{4}\left(2 n^{2}+1\right)\left(1-m c^{2}\right)^{-2}\left\{1+m\left(1+c^{2}-m c^{2}\right)^{2}\right\}\right]
\end{aligned}
$$

where

$$
F_{j}(x)=\pi^{-1 / 2} \int \exp \left\{-(y-x)^{2}-\frac{g}{n} y^{4}\right\} y^{j} d y
$$

\section{Appendix}

Evaluation of the constant in Eq. (2.25).

We will need the

Lemma. Let $F(X) \equiv F\left(x_{1}, \ldots, x_{n}\right)$ be a symmetric function of $x_{1}, \ldots, x_{n}$ and $\Delta(X)=$ $\prod_{1 \leqq i<j \leqq n}\left(x_{i}-x_{j}\right)$. Then for arbitrary numbers $\lambda_{i j}$ one has

$$
\int d X \Delta(X) F(X) \prod_{i<j}\left(x_{i}-x_{j}+\lambda_{i j}\right)=\int d X \Delta^{2}(X) F(X) .
$$

Proof. Expand the product $\prod_{i<j}\left(x_{i}-x_{j}+\lambda_{i j}\right)$ in powers of $x_{1}, \ldots, x_{n}$ and note that

$$
\int x_{1}^{\alpha_{1}} x_{2}^{\alpha_{2}} \ldots x_{n}^{\alpha_{2}} \Delta(X) F(X) d X=0
$$

if any two of the $\alpha_{i}$ are equal; this is so because if $\alpha_{i}=\alpha_{j}$, then integrand is antisymmetric in the variables $x_{i}$ and $x_{j}$. Therefore the monomial in $x_{1}, \ldots, x_{n}$ which will give a non-zero contribution to the integral must have all $\alpha_{i}$ distinct, and its degree is at least

$$
0+1+2+\ldots+n-1=\frac{1}{2} n(n-1) .
$$

This is also the degree of $\prod_{i<j}\left(x_{i}-x_{j}\right)=\Delta(X)$. Hence terms containing any $\lambda_{i j}$ drop out on integration. End of proof.

To calculate the constant in Eq. (2.25) we may choose

$$
V(A)=\operatorname{tr} A^{2}=\sum_{i=1}^{n} x_{i}^{2}
$$

Then

$$
\int \exp \left\{-\sum_{1}^{n}\left(x_{i}^{2}+y_{i}^{2}-2 c x_{i} y_{i}\right)\right\} \Delta(X) \Delta(Y) d X d Y
$$




$$
\begin{aligned}
& =\int \exp \left\{-\sum_{1}^{n}\left(\left(1-c^{2}\right) x_{i}^{2}+\left(y_{i}-c x_{i}\right)^{2}\right\} \Delta(X) \Delta(Y) d X d Y\right. \\
& =\int \exp \left\{-\sum_{1}^{n}\left(\left(1-c^{2}\right) x_{i}^{2}+y_{i}^{2}\right)\right\} \Delta(X) \Delta(Y+c X) d X d Y \\
& =c^{(1 / 2) n(n-1)} \int \exp \left\{-\left(1-c^{2}\right) \sum_{1}^{n} x_{i}^{2}\right\} \Delta^{2}(X) d X \int e^{-\sum_{1}^{n} y_{i}^{2}} d Y
\end{aligned}
$$

by the lemma. The integration over the $y_{i}$ is elementary. For that over $x_{i}$ change variables to

$$
x_{i}^{\prime}=\left(1-c^{2}\right)^{1 / 2} x_{i}
$$

so that

$$
\int \exp \left\{-\left(1-c^{2}\right) \sum_{1}^{n} x_{i}^{2}\right\} \Delta^{2}(X) d X=\left(1-c^{2}\right)^{-(1 / 2) n^{2}} \int e^{-\sum_{1}^{n} x_{i}^{2}} \Delta^{2}(X) d X .
$$

The last integral can be evaluated ${ }^{2}$ by introducing Hermite polynomials which are orthogonal for the gaussian weight. The final result is

$$
\begin{aligned}
\int \exp \{ & \left.-\Sigma\left(x_{i}^{2}+y_{i}^{2}-2 c x_{i} y_{i}\right)\right\} \Delta(X) \Delta(Y) d X d Y \\
= & c^{(1 / 2) n(n-1)} \pi^{(1 / 2) n}\left(1-c^{2}\right)^{-(1 / 2) n^{2}} n ! \prod_{0}^{n-1}\left(\pi^{1 / 2} 2^{-i} i !\right) \\
= & \pi^{n}\left(\frac{1}{2} c\right)^{(1 / 2) n(n-1)}\left(1-c^{2}\right)^{-(1 / 2) n^{2}} \prod_{1}^{n} i !
\end{aligned}
$$

For Eq. (5.6) we need to evaluate

$$
\begin{aligned}
& \int \sum_{1}^{n}\left(x_{i}^{4}+y_{i}^{4}\right) \exp \left\{-\sum_{1}^{n}\left(x_{i}^{2}+y_{i}^{2}-2 x_{i} y_{i}\right)\right\} \Delta(X) \Delta(Y) d X d Y \\
& =2 \int \sum_{1}^{n} x_{i}^{4} \exp \left\{-\sum_{1}^{n}\left(\left(1-c^{2}\right) x_{i}^{2}+y_{i}^{2}\right)\right\} \Delta(X) \Delta(c X) d X d Y \\
& =2 n c^{(1 / 2) n(n-1)}\left(1-c^{2}\right)^{-(1 / 2)\left(n^{2}+4\right)} \pi^{n / 2} \int \exp \left(-\sum_{1}^{n} x_{i}^{2}\right) x_{1}^{4} \Delta^{2}(X) d X
\end{aligned}
$$

as above. Once more introducing Hermite polynomials, the last integral is seen to $\mathrm{be}^{2}$

$$
\begin{aligned}
& \int \exp \left(-\sum_{1}^{n} x_{i}^{2}\right) x_{i}^{4} \Delta^{2}(X) d X \\
& =(n-1) ! \prod_{0}^{n-1}\left(2^{-i} i ! \pi^{1 / 2}\right) \sum_{i=0}^{n-1} \frac{\int x^{4} H_{i}^{2}(x) e^{-x^{2}} d x}{\int H_{i}^{2}(x) e^{-x^{2}} d x}
\end{aligned}
$$

2 [4], Chap. 6 
Finally, from the three term recurrence relation and orthogonality one gets

$$
\int x^{4} H_{i}^{2}(x) e^{-x^{2}} d x=\frac{3}{4}\left(2 i^{2}+2 i+1\right) \int H_{i}^{2}(x) e^{-x^{2}} d x .
$$

Putting everything together one sees that

$$
\begin{aligned}
& \int \sum_{1}^{n}\left(x_{i}^{4}+y_{i}^{4}\right) \exp \left\{-\sum_{1}^{n}\left(x_{i}^{2}+y_{i}^{2}-2 c x_{i} y_{i}\right)\right\} \Delta(X) \Delta(Y) d X d Y \\
& \quad=\left(1-c^{2}\right)^{-2}\left(n^{3}+\frac{1}{2} n\right) \int \exp \left\{-\sum_{1}^{n}\left(x_{i}^{2}+y_{i}^{2}-2 x_{i} y_{i}\right)\right\} \Delta(X) \Delta(Y) d X d Y,
\end{aligned}
$$

implying Eq. (5.6)

Acknowledgements. I am thankful to my colleagues, to C. Itzykson who got me interested in this problem, to J. B. Zuber who explained me with great patience their article and to M. Gaudin, J. des Cloizeaux, A. Gervois, G. Mahoux and J.M. Normand with whom I had many helpful discussions.

\section{References}

1. Brezin, E., Itzykson, C., Parisi, G., Zuber, J. B. : Commun. Math. Phys. 59; 35-51 (1978)

2. Bessis, D. : A new method in the combinatoric of the topological expansion, Commun. Math. Phys. 69, 147-163 (1979)

3. Itzykson, C., Zuber, J. B. : The planar approximation (II), J. Math. Phys. 21, 411-421 (1980)

4. Mehta, M. L. : Random matrices. Chap. 3. New York : Academic Press 1967

5. Morse, P.M.: Feshbach, H.: Methods of mathematical physics. Chap. 2.4. New York: McGrawHill 1953

6. Hardy, G. H., Littlewood, J. E., Polya, G. : Inequalities. p. 138. Cambridge: University Press 1964

7. Chadha S., Mahoux G., Mehta M. L.: A Method of integration over Matrix variables. II. J. Phys. A (in press)

8. Bessis D., Itzykson C., Zuber J. B., Adv. Appl. Math. 1, 109-157 (1980)

Communicated by E. Brézin

Received October 1, 1979 